\title{
PRODUÇÃo E DECOMPOSIÇÃO DE FOLHEDO EM UM TRECHO DE MATA ATLÂNTICA DE ENCOSTA NO MUNICIPIO DO GUARUJÁ, SP
}

\author{
Roberto Varjabedian (1) \\ Sergio Nereu Pagano (2)
}

\begin{abstract}
RESUMO - A produção de folhedo e a taxa de decomposição de folhedo (k) foram estimadas, no período de 2.8 .85 a 10.8 .86 , numa área de 1 ha de mata atlântica de encosta $\left(60^{\circ}\right.$ de inclinação e altitude de $140 \mathrm{~m}$ ), no município do Guarujá (Lat. S $24^{\circ} 16^{\prime}$; Long. W $46^{\circ} 19^{\prime}$ ), Estado de São Paulo. A queda pluviométrica anual média é de $2050 \mathrm{~mm}$ e não há estação seca definida. O solo é argilo-arenoso e ácido, com pH variando entre $3 \mathrm{e} 4$. A produção anual de folhedo foi de $7925 \mathrm{~kg} / \mathrm{ha}$. A fra- ção folhas contribuiu com $5040 \mathrm{~kg} /$ ha seguida pelas fraçōes ramos $(1950 \mathrm{~kg} / \mathrm{ha})$, flores $(491 \mathrm{~kg} / \mathrm{ha})$, frutos $(222 \mathrm{~kg} / \mathrm{ha})$ e material de origem animal $(222 \mathrm{~kg} / \mathrm{ha})$. A produção de folhedo e das fraçōes componentes foi contínua durante todo o ano. Os valores mensais de produção não revelaram nenhum padrão sazonal. A taxa de decomposição (k) foi estimada para condiçōes de equilíbrio dinâmico $(0,72)$ e também utilizando a porcentagem de peso remanescente da fração foliar $(0,83)$. $\mathrm{O}$ tempo médio para a decomposição de $50 \%$ do folhedo foi de 350 dias.
\end{abstract}

\begin{abstract}
Litter production and decomposition rate were studied in 1 ha of hillside atlantic forest ( $60^{\circ}$ inclination and $140 \mathrm{~m}$ high) located at Guarujá (24․ 16' S; 46․ 19' W) of São Paulo State from 2.8 .85 to 10.8 .86. This area have not a defined dry season and the annual average rainfall is $2050 \mathrm{~mm}$. The soil is classified as acidic and clay-sand with $\mathrm{pH} 3.0-4.0$. The annual litter production was estimated at $7925 \mathrm{~kg} / \mathrm{ha}$ : comprising leaves fraction contributing with $5040 \mathrm{~kg} / \mathrm{ha}$, followed by branches $(1950 \mathrm{~kg} / \mathrm{ha})$, flowers $(491 \mathrm{~kg} / \mathrm{ha})$, fruits $(222 \mathrm{~kg} / \mathrm{ha})$ and animal products $(222$ $\mathrm{kg} / \mathrm{ha}$ ). All fractions were produced during all over the year, without any detectable seazonal pattern. The decomposition constant $(k)$ was estimated for steady-state conditions $(0,72)$ and also utilizing the percentage of remaining weight of foliar fraction $(0,83)$. The time necessary for $50 \%$ of the litter decomposition was 350 days.
\end{abstract}

Key-words: Litterfall, decomposition, Coastal Rain Forest.

\section{Introdução}

Nas últimas duas décadas vários trabalhos foram realizados em ecossistemas naturais brasileiros enfocando ciclagem de nutrientes. Na região amazônica, Klinge e Rodrigues $(1968 a, b)$ estimaram a produção de folhedo e o conteúdo de nutrientes dessa produção em floresta de terra firme à nordeste de Manaus. O aspecto de produção de folhedo foi também abordado por Silva e Lobo (1982) com estudos realizados em floresta de terra firme, várzea e igapó nas proximidades de Belém do Pará e por Luizão (1982) que estudou a produção e decomposição de folhedo em floresta de terra firme na Amazônia Central. Para Mata Mesófila destacam-se os estudos de Meguro et al. (1979a,b; 1980) e Struffaldi-DeVuono e Marzolla (1984), na área metropolitana de São Paulo, dando atenção à análise da influência antrópica exercida sobre a dinâmica dos nutrientes e o processo de decomposição e de Pagano (1985), no município de Rio Claro, estudando a transferência de nutrientes da biomassa para o solo através da produção e decomposição de folhedo.

(1) Bacharel em ciências biológicas. Caixa Postal 0161/CEP 01051, SP.

(2) Departamento de Botânica, Instituto de Biociências, UNESP, Rio Claro. Caixa Postal 178, Estado de São Paulo. 
Em Mata Ciliar, no interior do Estado de São Paulo, evidenciam-se os trabalhos de Carpanezzi (1980) e Delitti (1984), abordando aspectos de ciclagem de nutrientes, comparando esse ecossistema natural e ecossistemas implantados.

O Cerrado possui também um considerável número de estudos dentre os quais se destacam o de Silva (1983) sobre produção e decomposição de folhedo em uma área de cerrado em Brasilia (DF) e os de Coutinho (1979, 1980 e 1982) e Pivello-Pompéia (1985) sobre a influência do fogo sobre a dinâmica dos nutrientes, no município de Pirassununga, SP.

Em relação a Floresta Pluvial Tropical da zona costeira, considerada área de risco para preservação, existem pouquíssimos estudos que dão ênfase a aspectos dinâmicos.

Tendo em vista a velocidade com que essa vegetação está sendo destruída e a necessidade de conhecimento dos parâmetros básicos que regem sua dinâmica, o objetivo deste trabalho foi o de estimar a produção mensal e anual de folhedo e a taxa de decomposição desse folhedo em um trecho de Mata Atlântica de encosta, no município do Guarujá, SP.

\section{Material e Métodos}

Localização - O ecossistema estudado localiza-se em uma encosta do Morro do Icanhema, situado no sudoeste da Ilha de Santo Amaro, município do Guarujá (Lat. S $24^{\circ}$ 16'; Long. W 46 $19^{\prime}$ ), no litoral do Estado de São Paulo.

Delimitou-se uma área de 1 ha de encosta, cuja extensão desde a base até o topo, é cerca de $170 \mathrm{~m}$. O experimento foi montado acima da faixa de $70 \mathrm{~m}$ para evitar a influência antrópica uma vez que as planícies circundantes são urbanizadas. A inclinação é de $60^{\circ} \mathrm{e}$ a altitude de $140 \mathrm{~m}$.

Clima - Os dados de clima foram obtidos no $7^{\circ}$ Distrito de Meteorologia do Ministério da Agricultura, localizado a $3 \mathrm{~km}$ ao norte da áren estudada, no município de Santos.

Solo - Para análise química e física do solo foram coletadas 5 amostras em 5 diferentes níveis da encosta, dentro da área delimitada para estudo, sendo que cada nível distava $20 \mathrm{~m}$ do seguinte, a partir do topo em direção à base. Cada amostra foi constituída da mistura de 3 sub-amostras coletadas em pontos diferentes em cada nível. As perfurações foram feitas desde a superfície até $20 \mathrm{~cm}$, coletando-se todo o solo até essa profundidade. Uma alíquota de cada amostra foi separada para análise química realizada segundo Raij e Quaggio (1983) e para análise granulométrica pelo método da pipeta (Medina, 1972).

Produção de folhedo - Para verificação da quantidade de folhedo produzido foram utilizados 10 coletores quadrados de madeira, $50 \mathrm{~cm} \times 50 \mathrm{~cm} \times 15 \mathrm{~cm}$, com fundo de tela de nailon de malha de $1 \mathrm{~mm}$, a $30 \mathrm{~cm}$ da superfície do solo. Em cada lado de uma trilha central de $100 \mathrm{~m}$, ascendente na encosta, foram distribuídos 5 coletores, cada qual distando $20 \mathrm{~m}$ do coletor seguinte, enquanto que a distância entre a trilha central e cada um dos coletores foi de $30 \mathrm{~m}$. Mensalmente, de 2.8.85 a 10.8.86, o material depositado em cada coletor fo coletado, seco em estufa a $80^{\circ} \mathrm{C}$ e separado em frações: folhas, ramos até $2 \mathrm{~cm}$ de diâme. tro (Proctor 1983), flores, frutos e material de origem animal, obtendo-se o peso de cad́ fração e o peso total. Para cada mês calculou-se a média e o desvio-padrão para os pesos totais dos 10 coletores. A verificação de diferenças significativas $(\alpha=0,05)$ entre as mé dias mensais foi feita através do teste não paramétrico de Kruskal-Wallis com compara ções múltiplas (Campos 1983). A produção anual foi estimada pela soma das médias men sais e expressa em $\mathrm{kg} / \mathrm{ha}$.

Folhedo-Acumulado - A estimativa da quantidade de folhedo presente sobre : superfície do solo, foi determinada através da coleta mensal de 5 amostras obtidas pek lançamento aleatório de uma armação quadrada de $50 \mathrm{~cm} \times 50 \mathrm{~cm}$, colhendo-se todo folhe do presente nessa área. Para cada mês, de 2.8,85 a 10.8.86, calculou-se a média e o des 
vio-padrão para os pesos totais das 5 amostras após secá-las separadamente em estufa a $80^{\circ} \mathrm{C}$ até peso constante. A média anual da quantidade de folhedo presente sobre a superfície do solo foi obtida calculando-se a média das médias mensais, e os valores são expressos em kg/ha.

Taxa de decomposição e tempo médio de renovação - A taxa de decomposição de folhedo (k) foi obtida através da equação de Olson (1963) adequada para situaçōes de equilíbrio dinâmico:

$$
K=\frac{L}{\text { Xss ("steady-state") }}
$$

onde: $L=$ quantidade de folhedo produzido anualmente.

Xss = média anual de quantidade de folhedo acumulado sobre a superfície do solo.

$\mathrm{O}$ coeficiente $\mathrm{K}$ foi também calculado para a perda de peso da fração foliar segundo Jenny et al. (1949) e Olson (1963):

$$
K=-\ln \left(1-k^{\prime}\right)
$$

sendo que k' pode ser obtido através da equação:

$$
K^{\prime}=\frac{\Delta x}{x_{0}}
$$

onde: $\Delta \mathrm{x}=$ quantidade de material perdido no tempo $\Delta \mathrm{t}$.

$\mathrm{XO}=$ quantidade inicial do material.

Para determinação de k' e consequentemente de k foram utilizadas 40 bolsas de tela de nailon de $20 \mathrm{~cm} \times 10 \mathrm{~cm}$, com malha de $2 \mathrm{~mm}$. As bolsas contendo $10 \mathrm{~g}$ de folhas recém caidas de diversas espécies, coletadas em vários pontos da área estudada, foram distribuídas aleatoriamente sobre a superfície do solo da mata. Durante o período de estudo foram realizadas 4 coletas com intervalos de 3 meses, retirando-se 10 bolsas por vez. No laboratório, o conteúdo de cada bolsa foi seco separadamente em estufa a $80^{\circ} \mathrm{C}$ e posteriormente pesado. A média e o desvio-padrão foram calculados para o conteúdo das 10 bolsas, obtendo-se a porcentagem média de perda de peso no tempo considerado. son (1963):

O tempo médio de renovação para o folhedo foi calculado segundo a equação de Ol-

$$
T_{50 \%}=\frac{0,6931}{k \text { ("steady-state") }}
$$

\section{Resultados}

Clima - Segundo a classificação climática de Koeppen (1948) o clima da regiāo está classificado como Af: clima tropical chuvoso (A), com chuvas o ano todo (f) e segundo Nimer (1977) é do tipo Sup-U (Super úmido, sem seca). A queda pluviométrica anual atinge em média $2050 \mathrm{~mm}$, ocorrendo variaçōes entre 1500 e $2500 \mathrm{~mm}$. As Figuras 1 e 2 mostram, respectivamente, as variações mensais para o período de experimento e também para um período de 10 anos. Verificou-se que a queda pluviométrica é geralmente mais intensa nos meses de verão. As oscilações inesperadas são frequentes, embora a 
Figura 2 que representa dados médios, aparente um regime definido. A temperatura teve um comportamento mais uniforme com valores maiores nos mesès de verão e menores nos meses de inverno.

Solo - Os resultados da análise granulométrica e química são apresentadas nas Tabelas 1 e 2 . As amostras aparecem na sequência em que foram coletadas, desde o topo (1) até próximo à base (5). A composição granulométrica caracterizou um solo argilo-arenoso e as amostras tiveram nesse aspecto características semelhantes (Tabela 1). A análise química revelou um solo ácido com $\mathrm{pH}$ variando entre 3,1 e 3,9. A porcentagem de matéria orgânica do solo variou entre 5,31 e 6,88. Em linhas gerais os teores de $\mathrm{K}^{+} \mathrm{e} \mathrm{Mg}^{2+}$ foram considerados médios enquanto que o teor de $\mathrm{P}$ foi considerado baixo. Os valores encontrados para acidez potencial do solo $\left(\mathrm{H}^{+}+\mathrm{Al}^{3+}\right)$ foram considerados altos enquanto que os valores para porcentagem de saturação de bases (V) foram considerados baixos (Tabela 2).

TABELA 1 - Composição granulométrica do solo. Valores em porcentagem de terra fina seca ao ar. As amplitudes das classes de diâmetro são dadas entre parênteses em milimetros.

\begin{tabular}{cccccc}
\hline \multicolumn{7}{c}{ Amostra $n^{\circ}$} & \multicolumn{3}{c}{} \\
\hline Identificação & Areia Grossa & Areia Fina & Areia & Silte & Argila \\
\hline & $(2: 0,25)$ & $(0,25: 0,05)$ & $(2-0,05)$ & $(0,05-0,002)$ & $(<0,002)$ \\
\hline 01 & 49,1 & 5,3 & 54,4 & 15,0 & 30,6 \\
02 & 37,7 & 7,7 & 45,4 & 16,9 & 37,7 \\
03 & 43,1 & 6,2 & 49,3 & 17,0 & 33,7 \\
04 & 37,7 & 5,2 & 42,9 & 21,3 & 35,8 \\
05 & 35,3 & 8,7 & 44,0 & 21,7 & 34,3 \\
\hline
\end{tabular}

Produção de folhedo - A produção anual de folhedo foi de $7925 \mathrm{~kg} / \mathrm{ha}$ sendo que a fração folhas participou com a maior porcentagem $(63,6 \%)$, seguida pelas frações ramos $(24,6 \%)$, flores $(6,2 \%)$, frutos $(2,8 \%)$ e material de origem animal $(2,8 \%)$. Os valores médios para a produção mensal, juntamente com os valores médios para cada fração são representados na Figura 3. A fração folhas apresentou os mais altos valores nos meses de janeiro, fevereiro e outuḅro, não ocorrendo sazonalidade marcante. As demais fraçōes mostraram-se variáveis sendo que os maiores valores para as frações ramos, flores e frutos ocorreram respectivamente nos meses de abril, maio e dezembro. Para o material de origem animal os valores mantiveram-se semelhantes de outubro a abril decaindo nos demais meses. As médias mensais e os respectivos desvios-padrão relativos à produção de folhedo são representados, em $\mathrm{kg} / \mathrm{ha}$, na Figura 4 . Os meses de janeiro, fevereiro e abril apresentaram os mais altos valores enquanto que os meses de junho, julho e setembro ficaram com os menores. Os desvios-padrão caracterizaram a variação entre os pontos de localização dos coletores.

O teste não paramétrico de Kruskal-Wallis com comparaçōes múltiplas revelou diferenças significativas a nível de $5 \%(\alpha=0,05)$ somente entre os meses de setembro e fevereiro, setembro e março, junho e fevereiro e julho e fevereiro.

Folhedo acumulado - A Figura 5 mostra os valores mensais médios estimados em $\mathrm{kg} / \mathrm{ha}$, e os respectivos desvios-padrão para a quantidade de folhedo presente sobre 0 solo na área estudada. Os dados mostram a ocorrência de oscilações no estoque desse folhedo no decorrer do ano enquanto que a irregularidade de distribuição do folhedo sobre a superfície do solo pode ser evidenciada pelos altos valores dos desvios-padrão. A média anual estimada foi de $10.904,72 \pm 2642 \mathrm{~kg} / \mathrm{ha}$. 
TABELA 2 - Resultados da análise química do solo. $\mathrm{S}=$ soma de bases $\left(\mathrm{Ca}^{+2}+\mathrm{Mg}^{+2}+\mathrm{K}^{+}\right)$; $\mathrm{T}=$ capacidade de troca catiónica $=\mathrm{S}+\left(\mathrm{H}^{+}+\mathrm{Al}^{3+}\right)$ e V $=$ porcentagem de saturação de bases $(100 \mathrm{~S} / \mathrm{T})$.

\begin{tabular}{|c|c|c|c|c|c|c|c|c|c|c|}
\hline \multirow{2}{*}{$\frac{\text { Amostra no }}{\text { Identificaçăo }}$} & \multirow{2}{*}{$\begin{array}{c}\mathrm{pH} \\
\mathrm{CaCl}_{2}\end{array}$} & \multirow{2}{*}{$\begin{array}{c}\text { Matéria } \\
\text { Orgânica } \\
\%\end{array}$} & \multirow{2}{*}{$\begin{array}{c}P \\
\text { resina } \\
\mathrm{ug} / \mathrm{cm}^{3}\end{array}$} & $\mathrm{~K}^{+}$ & $\mathrm{Ca}^{+2}$ & $\mathrm{Mg}^{+2}$ & $\left(\mathrm{H}^{+}+\mathrm{Al}^{3+}\right)$ & $\mathrm{s}$ & $T$ & \multirow[t]{2}{*}{$\vee \%$} \\
\hline & & & & \multicolumn{6}{|c|}{$\mathrm{meq} / 100 \mathrm{~cm}^{3}$} & \\
\hline 01 & 3,1 & 5,86 & 9,3 & 0,17 & 0,55 & 0,32 & 31,37 & 1,04 & 32,41 & 3,0 \\
\hline 02 & 3,3 & 5,77 & 8,9 & 0,27 & 0,57 & 0,32 & 25,33 & 1,16 & 26,49 & 4,0 \\
\hline 03 & 3,4 & 5,31 & 9,7 & 0,27 & 0,53 & 0,38 & 25,33 & 1,18 & 26,51 & 4,0 \\
\hline 04 & 3,2 & 6,88 & 8,9 & 0,23 & 0,45 & 0,42 & 28,15 & 1,10 & 29,25 & 4,0 \\
\hline 05 & 3,9 & 6,60 & 14,8 & 0,34 & 2,70 & 2,01 & 14,96 & 5,05 & 20,01 & 25,0 \\
\hline
\end{tabular}

Taxa de decomposição e tempo médio de renovação - $O$ valor da taxa de decomposição do folhedo $(K)$ para condiçōes de equilibrio dinâmico foi de 0,72 . Os coeficientes $K^{\prime}$ e $\mathrm{K}$ para perda da fração foliar em 1 ano foram respectivamente 0,56 e 0,83. O tempo médio de renovação foi de 0,96 que corresponde a 350 dias. A porcentagem média de perda de peso nas bolsas de nailon, em relação ao tempo, é representada na Figura 6 que ilustra apenas as tendências gerais do processo.

TABELA 3 - Produçăo total de folhedo, contribuiçăo da fraçăo foliar e taxa de decomposiçăo (K) de ecossistemas tropicais.

\begin{tabular}{lcccc}
\hline & FOLHEDO & FRAÇĀO & & \\
PLORESTAMOCAL & $\begin{array}{c}\text { PRODUZIDO FOLIAR } \\
\text { (Kg/ha/ano) }\end{array}$ & (kg/ha/ano) & & FONTE \\
\hline
\end{tabular}

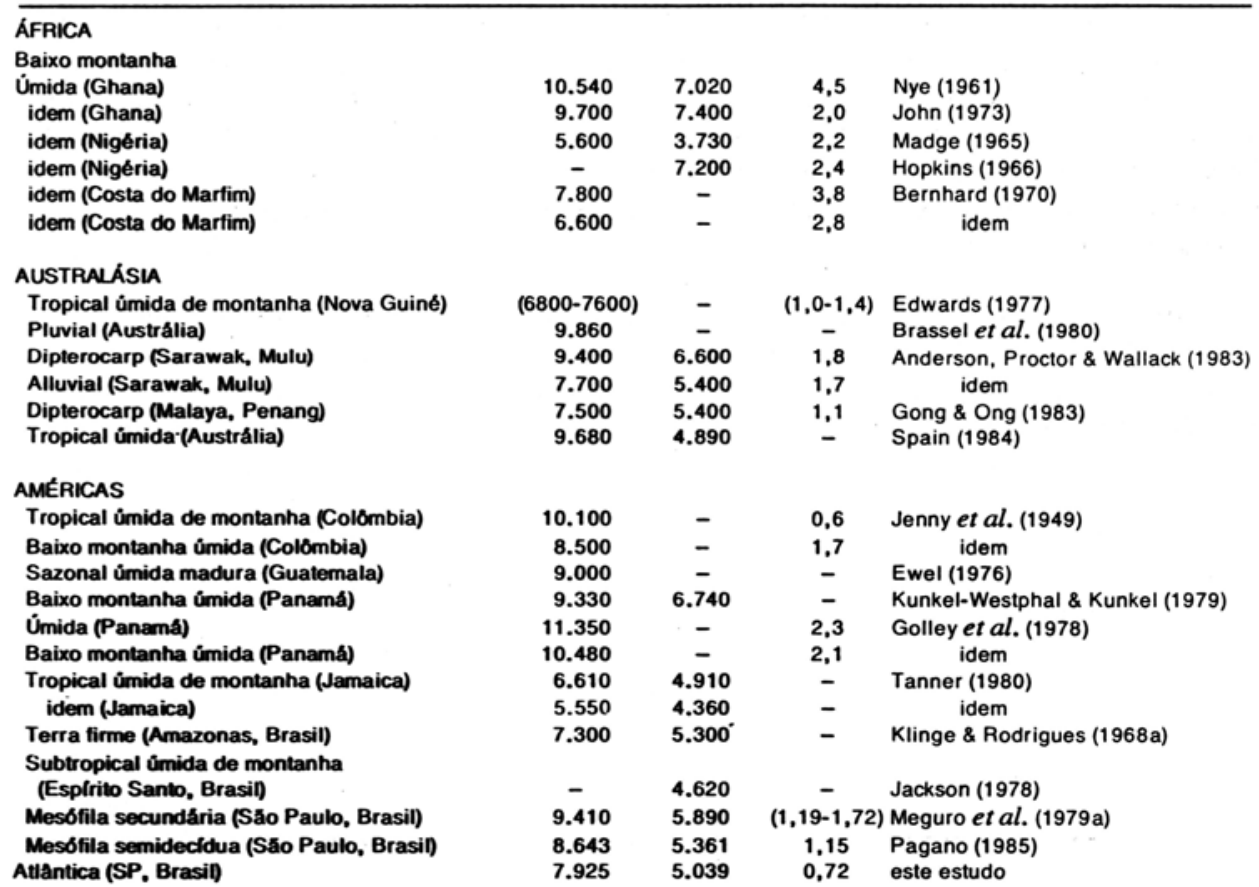




\section{Discussão}

O resultado obtido neste estudo para a produção anual de folhedo foi de $7925 \mathrm{~kg} / \mathrm{ha}$, valor que situa-se em uma faixa intermediária quando comparado a valores encontrados em florestas tropicais no Brasil e em todo o mundo (Tabela 3). A produção de folhedo e das fraçōes componentes foi contínua durante todo $o$ ano sendo que as variações observadas não revelaram nenhum padrão sázonal. Apenas a fração de material de origem animal apresentou valores semelhantes entre outubro e abril, fato que pode estar relacionado a maior atividade dos organismos em geral nesse período. As variações relativas à produção de folhedo ocorrida entre os meses e a diferença entre os valores para os 10 coletores em cada mês, embora caracterizem a diversidade e a heterogeneidade de distribuição das espécies na área estudada, sugerem a necessidade de utilização de um número maior de coletores no sentido de reduzir o valor dos desvios-padrão encontrados em relação às médias, conforme já mencionado por diversos autores, dentre eles Newbould (1967), Klinge e Rodrigues (1968a) e Proctor (1983). Apesar de apresentar essas variações, a queda de folhedo mostrou-se contínua, com poucas diferenças significativas, sendo que em 8 meses do período de estudo os valores médios superaram $600 \mathrm{~kg} / \mathrm{ha}$.

Embora os valores de produção mensal, de folhedo total e da fração foliar, sejam evidenciados para a maioria dos estudos em florestas tropicais com o pico máximo na estação seca, este fato não foi observado neste estudo, ocorrendo uma leve tendência geral no sentido inverso. Esse comportamento é semelhante ao encontrado por Jackson (1978) para fração foliar, em mata subtropical úmida, no Espírito Santo, sendo que nessa região a estação seca é melhor definida. Meguro et al. (1979a) também não observaram sazonalidade marcante em mata mesófila secundária na cidade de São Paulo, embora tenham ressaltado que esse fato, como no caso do presente estudo, possa ser decorrência do curto periodo de experimento.

As oscilações no regime pluviométrico, principalmente aquelas relativas aos valores mensais, são provavelmente acentuados pela proximidade do oceano e tornam difícil a delimitação de estações mais ou menos chuvosas. O mês de julho, por exemplo, embora seja geralmente menos chuvoso, apresentou o terceiro maior valor de pluviosidade enquanto que o mês de outubro, geralmente mais chuvoso, ficou com menor valor registrado. Desta forma, como não foram verificadas influências visíveis é possivel que, em termos imediatos, haja independência entre a intensidade da queda pluviométrica e a produção mensal de folhedo, fato esse constatado por Pagano (1985) em mata mesófila semidecídua.

No que diz respeito ao folhedo acumulado, os valores médios variaram entre 7500 $\mathrm{kg} / \mathrm{ha}$ (mínimo) e $15.500 \mathrm{~kg} / \mathrm{ha}$ (máximo), respectivamente em agosto e janeiro, sendo considerados altos quando comparados a maioria dos ecossistemas tropicais. As maiores variaçōes mensais são decorrentes da disposição horizontal das diferentes espécies que constituem a fitocenose, que caracteriza-se por apresentar alta diversidade específica. Outras razōes possiveis seriam a retenção de folhedo pelas raízes tabulares que funcionam como obstáculos em dias de chuva muito intensa e a queda de árvores senescentes ou mortas que é auxiliada pelos ventos marinhos, às vezes fortes.

A escolha do cálculo do coeficiente de decomposição (K) para condiçōes de equilibrio dinâmico foi feita pela sua ampla utilização em diversos estudos, o que facilita as comparaçōes. Desta forma, o valor de 0,72 estimado para este estudo é menor do que aqueles estimados para muitos ecossistemas tropicais (Tabela 3 ) onde $K>1$ (Olson 1963).

$\mathrm{O}$ valor estimado para o coeficiente de decomposição $(K)$ para perda de peso da fração foliar, que foi de 0,83 , mostrou-se próximo do valor encontrado para condições de equilibrio dinâmico, reafirmando a lentidão do processo de decomposição. A diferença encontrada entre os coeficientes, calculados de modo diferente, pode estar relacionada ao fato de que o valor maior $(0,83)$ refere-se apenas a decomposição da fração foliar e não do 
folhedo como um todo (que inclui as outras frações) sendo provável que desta forma ela tenha sido mais rápida. O tempo médio de renovação, estimado em 350 dias, foi próximo do encontrado para fração foliar nas bolsas de nailon, que em um ano de experimento perdeu em média $56,4 \%$ do seu peso inicial.

O baixo valor da taxa de decomposição pode provavelmente estar relacionado, entre outros fatores, ao baixo índice pluviométrico ocorrido nos seis primeiros meses do experimento. Além disso, segundo Sanchez (1976 apud Anderson £ Swift 1983), os valores encontrados para o $\mathrm{pH}$ e acidez potencial do solo $\left(\mathrm{H}^{+}+\mathrm{Al}^{3+}\right)$ poderiam estar influenciando negativamente a atividade dos microrganismos decompositores.

\section{Agradecimento}

Ao Prof. Dr. Francisco de A.F. de Mello, do Departamento de Solos, Geologia e Fertilizantes, ESALQ, pela análise das amostras de solo.

\section{Referências Bibliográficas}

ANDERSON, J.M.; PROCTOR, J. £ WALLACK, H.W. 1983. Ecological studies in four contrasting areas of lowland rain forests in Gunung Mulu National Park. III. Decomposition procesces and nutrient losses from leaf litter. J.Ecol 71: 503-528.

ANDERSON, J.M. $£$ SWIFT, M.J. 1983. Decomposition in tropical forests. In: Tropical Rain Forest: Ecology and Management. S.L. Sutton. T.C. Whitmores £ A.C. Chadwick (eds.). Special Publications Series of the British Ecological Society no 2, p. 287-309.

BERNHARD, F. 1970. Étude de la litière et sa contribution an cycle des eleménts mireráux en forêt ombrophile de Côte d'Ivoire. Oecologia Pl. S: 247-266.

BRASSEL, H.M.; UNWIN, G.L. £ STOCKER, G.C. 1980. The quantity, temporal distribution and mineral-element content of litterfall in two forest types at two sites in Tropical Australia. Ecology 68: 123-139.

CAMPOS, H. 1983. Estatística Experimental Não-paramétrica. Departamento de Matemática e Estatística da Escola Superior de Agricultura “'Luis de Queiroz", Univ. S. Paulo, Piracicaba.

CARPANEZZI, A.A. 1980. Deposiçâo de material orgânico e nutrientes em uma floresta natural e em uma plantação de eucaliptos no interior do Estado de São Paulo. Dissertação de Mestrado, Escola Superior de Agricultura "Luis de Queiroz", Univ. S. Paulo, Piracicaba.

COUTINHO, L.M. 1979. Aspectos ecológicos do fogo no cerrado. III. A precipitação atmosférica de nutrientes minerais. Revta. bras. Bot. 2: $97-101$.

COUTINHO, L.M. 1980. As queimadas e seu papel ecológico Bras. Florestal 44: 7-23.

COUTINHO, L.M. 1982. Ecological effects of fire in Brazilian Cerrado. Ecological Studies 42. In: Ecology of Tropical Savannas. Springer-Verlag. Berlin, p. 273-291.

DELITTI, W.B.C. 1984. Aspectos comparativos da ciclagem de nutrientes minerais na mata ciliar, no campo cerrado e na floresta implantada de Pinus elliotti Engebm. Var. elliottie (Mogi-Guaçu, SP). Tese de Doutorado, Instituto de Biociências, Univ. S. Paulo.

EDUARDS, P.J. 1977. Studies of Mineral cycling in a Montane Rain Forest in New Guinea. J. Ecol. 65: 971-992.

EWEL, J.J. 1976. Litter fall and Leaf Decomposition in a Tropical Forest Sucession in Eastern Guatemala.J.Ecol. 64: 293-308.

GOLLEY, F.B.; Mc GINNIS, J.T.; CLEMENTS, R.G.; CHILD, G.I. £ DUEVER, M.J. 1978. Ciclagem de minerais em um ecossistema de floresta tropical úmida (Tradução) E.P.U - EDUSP, S. PAULO.

GONG, W.K: $£$ ONG, J.E. 1983. Litter production and decomposition in a coastal hill dipterocarp forest. In: Tropical Rain Forest: Ecology and Management. S.L. Sutton; T.C. Whitmore $£$ A.C. Chadwick (eds.). Special Publications series of the British Ecological Society no 2, p. 275-285.

HOPKINS, B. 1966. Vegetation of the Olokemeji Forest Reserve. NIGERIA. IV. The litter and soil with special reference to their seasonal changes. J.Ecol. 54: 687-703.

JACKSON, J.F. 1978. Seasonality of Flowering and Leaf-Fall in a Braziliar Subtropical lower Montane Moist Forest. Biotropica 10: 38-42.

JENNY, H.; GESSEL, S.P. $£$ BINGHAM, F.T. 1949. Comparative study of decomposition rates of organic matter in temperature and tropical regions. Soil. Sci. 68: 419-432.

JOHN, D.M. 1973. Accumulation and Decay of Litter and Net Production of Forestin Tropical West África. Oikos 24: 430-435.

KLINGE, H. $£$ RODRIGUES, W.A. 1968a. Litter Production in an Area of Amazonian Terra Firme Forest. Part I. Litter-fall Organic Carbon and Total Nitrogen Contens of Litter. Amazoniana 1: 287-302.

KLINGE, H. £ RODRIGUES, W.A. 1968b. Litter Production in an Area of Amazonian Terra Firme Forest. Part II. Mineral Nutrient Content of the Litter. Amazoniana 1: 303-310.

KOEPPEN, W. 1948. Climatologia. Fondo de Cultura Econômica. México-Buenos Aires.

KUNKEL-WESTPHAL, J. £ KUNKEL, P. 1979. Litter Fall in a Guatemalan Primary Forest With Details of Leaf-Shedding by some common tree species. J. Ecol 67: 665-680. 
LUIZĀO, F.J. 1982. Prödução e decomposição da liteira em floresta de terra firme na Amazónia Central. Aspectos químicos e biológicos da lixiviação e remoção de nutrientes da liteira. Dissertação de Mestrado. Instituto de Pesquisas da Amazônia. Fundaçāo Universidade do Amazonas.

MADGE, D.S. 1965. Litter fall and litter disappearance in a tropical forest. Pedobiologia 5: 273-288.

MEDINA, H.P. 1972. Física do Solo: Constituição física: In: Elementos de Pedologia, Coordenado por Antônio £ Moniz. Edusp £ Editora Polígono, S. Paulo, p. 11-20.

MEGURO, M.; VINUEZA, G.N. £ DELITTI, W.B.C. 1979a. Ciclagem de nutrientes minerais na mata mesófila secundária - São Paulo. I. Produção e conteúdo de nutrientes minerais no folhedo. Bolm Botânica Univ. S. Paulo 7: 11-31.

MEGURO, M.; VINUEZA, G.N. $£$ DELITTI, W.B.C. 1979b. Ciclagem de nutrientes minerais na mata mesófita secundária - São Paulo. II. O papel da precipitação na importaçāo e transferência de potássio e fósforo. Bolm Botânica Univ. S. Paulo. 7:61-67.

MEGURO, M.; VINUEZA, G.N. £ DELITTI, W.B.C. 1980. Ciclagem de nutrientes minerais na mata mesófila secundária - São Paulo. III. Decomposiçāo do material foliar e liberaçāo dos nutrientes minerais. Bolm Botânica Univ. S. Paulo 8: 7-20.

NEWBOULD, P.J. 196?. Methods of Estimating the Primary Production of Forests. IBP Handbook no 2. Blackwell Scientific Publications, Ox ford.

NIMER, E. 1977. Clima. In: Fundação Instituto Brasileiro de Geografia e Estatística ed. Geografia do Brasil; Rio de Janeiro, IBGE. v.1, p. 39-58; v.2, p. 47-84; v.3, p. 38-58; v.4, p. 51-89; v.5, p. 35-79.

NYE, P.H. 1961. Organic Matter and Nutrient Cycles Under Moist Tropical Forest. Plant Soil XIII (4): 333-346.

OLSON, J.S. 1963. Energy storage and the balance of producers and decomposers in ecological systems. Ecology 44: 322-331.

PAGANO, S.N. 1985. Estudo florístico, fitossociologico e de ciclagem de nutrientes em Mata Mesófila Semidecidua, no município de Rio Claro. Tese de livre docência, Instituto de Biociências, UNESP - Rio Claro - S.P.

PIVELLO-POMPÉIA, V.R. 1985. Exportaçâo de macronutrientes para a atmosfera durante queimadas realizadas no campo cerrado de Emas, Pirassununga, S.P. Dissertação de Mestrado, Instituto de Biociências, Univ. S. Paulo.

PROCTOR, J. 1983. Tropical forest litterfall. I. Problems of data comparision In: Tropical Rain Forest: Ecology and Management. S.L. Sutton; T.C. Whitmore $£$ A.C. Chadwick (eds.). Special Publications Series of the Britsh Ecological Society $n=2$, p. 267-273.

RAIJ, B.V. $£$ QUAGGIO, J.A. 1983. Métodos de análise de solo para fins de fertilidade. Bolm Técnico $n^{o}$ 81, Instituto Agronômico, Campinas.

SILVA, J.S. 1983. Alguns aspectos da ciclagem de nutrientes em uma área de cerrado (Brasfia, DF): Chuva, produção e decomposição de liter. Dissertação de Mestrado, Univ. Brasília.

SILVA, M.F.F. $£$ LOBO, M.G.A. 1982. Nota sobre deposição de matéria orgânica em floresta de terra firme, várzea e igapó. Bolm Mus. Pará. "Emilio Goeldi", Ser. Botânica 56: 1-13.

SPAIN, V.A. 1984. Litter Fall and the standing crop of litter in three tropical australian rainforest. J.Ecol. 72: 947-961.

STRUFFALDI-DE-VUONO, Y. £ MARZOLLA, M.C. 1984. Decomposição ou serapilheira nas proximidades de uma siderúrgica. Anais IV Congresso da SBSP: 83-86.

TANNER; E.V.J. 1980. Litter fall in montane rain forests of Jamaica and its relation to climate. J. Ecol. 68: 833-848. 

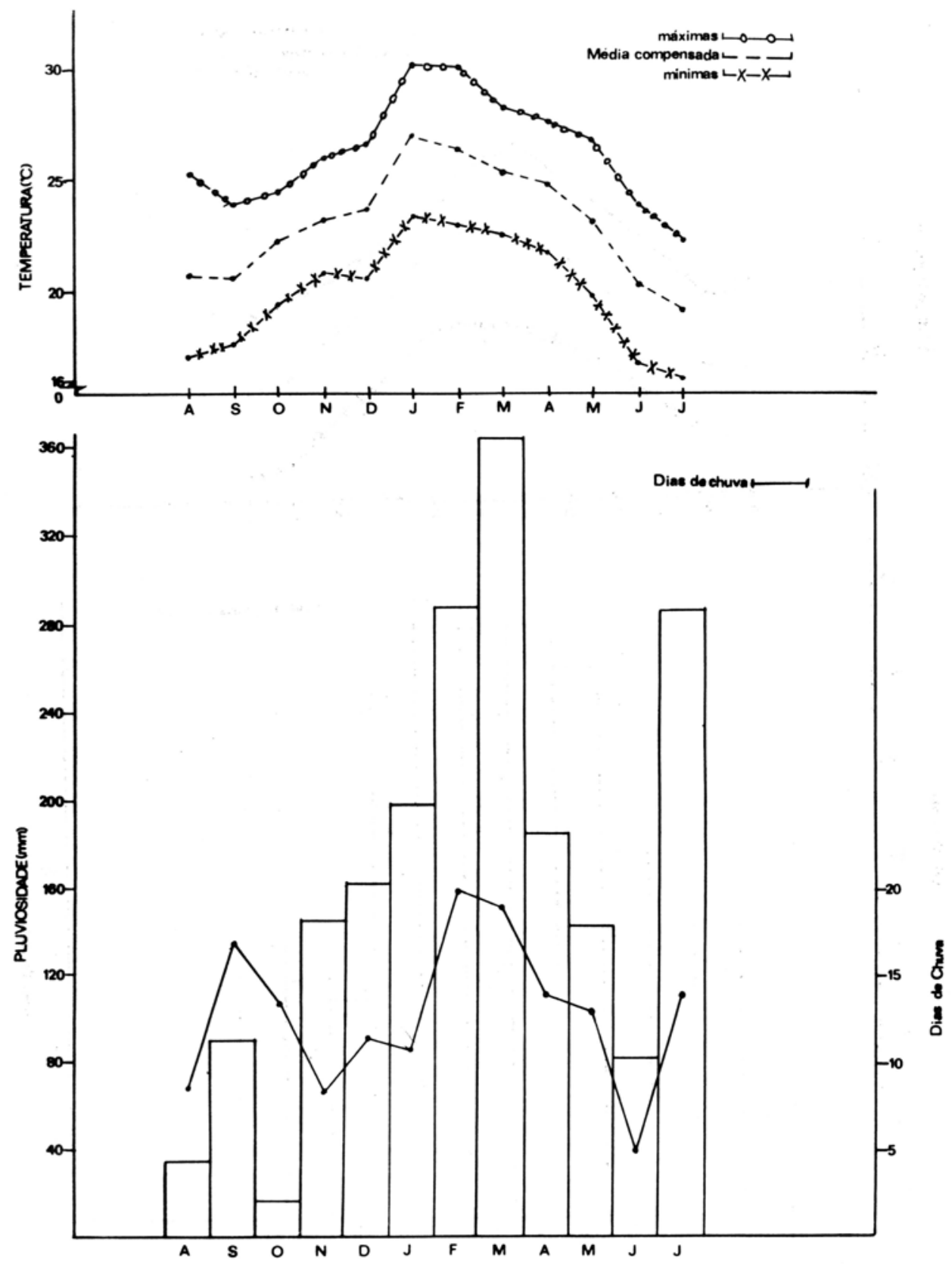

Fig. 1 - Variaçōes climáticas para o período de estudo (1985/1986). 

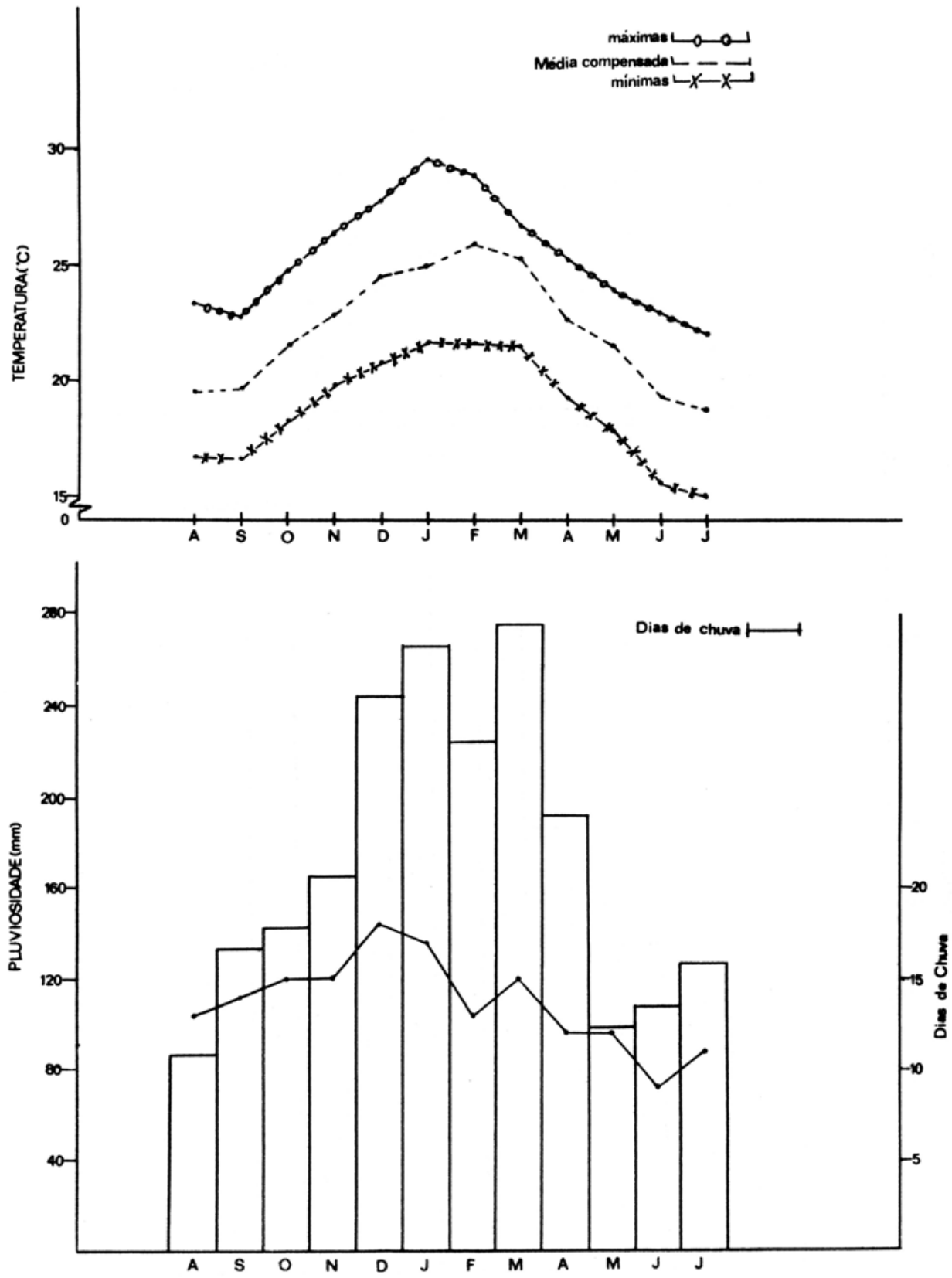

Fig. 2 - Variaçōes climáticas para um perfodo de 10 anos (1976/1985). 

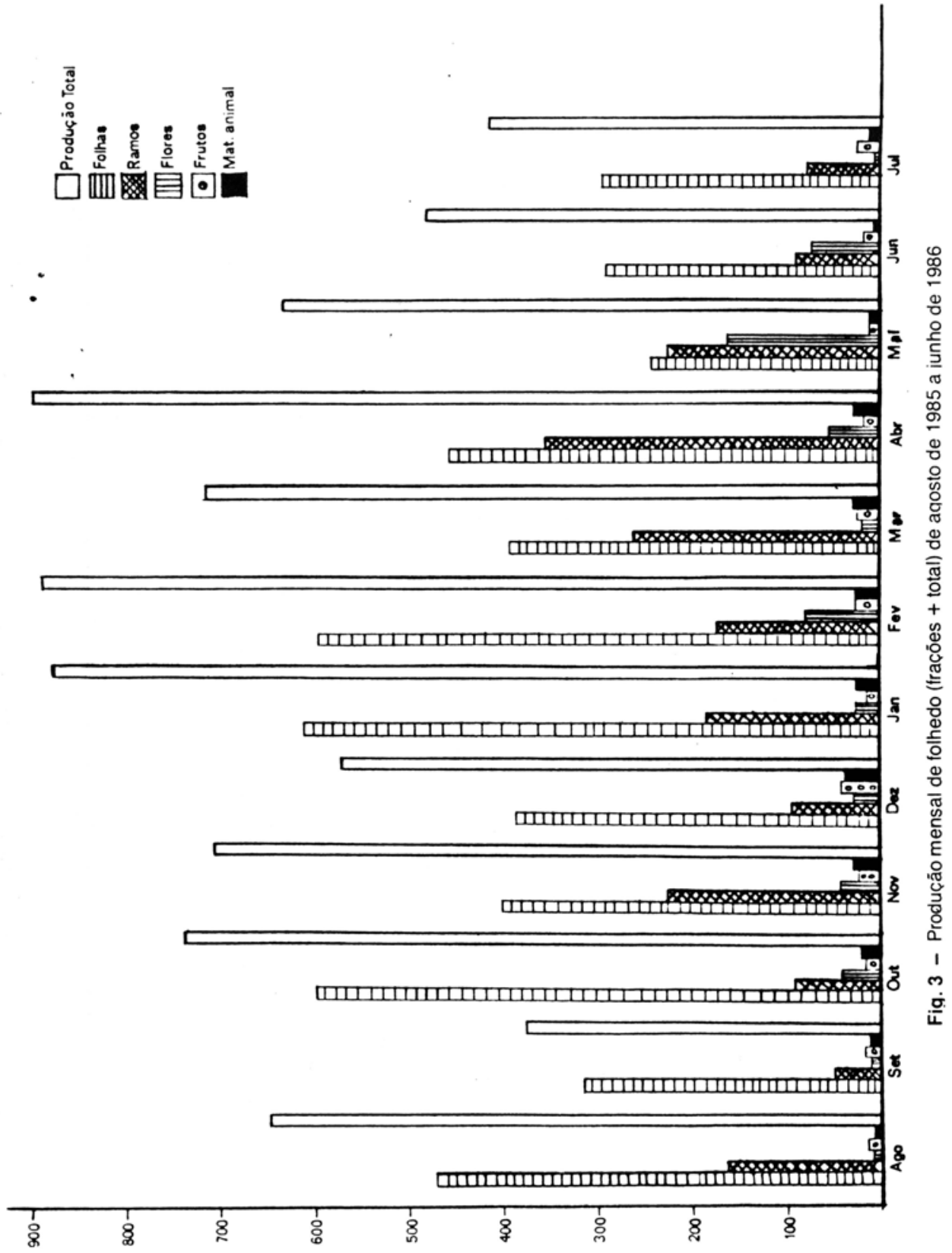


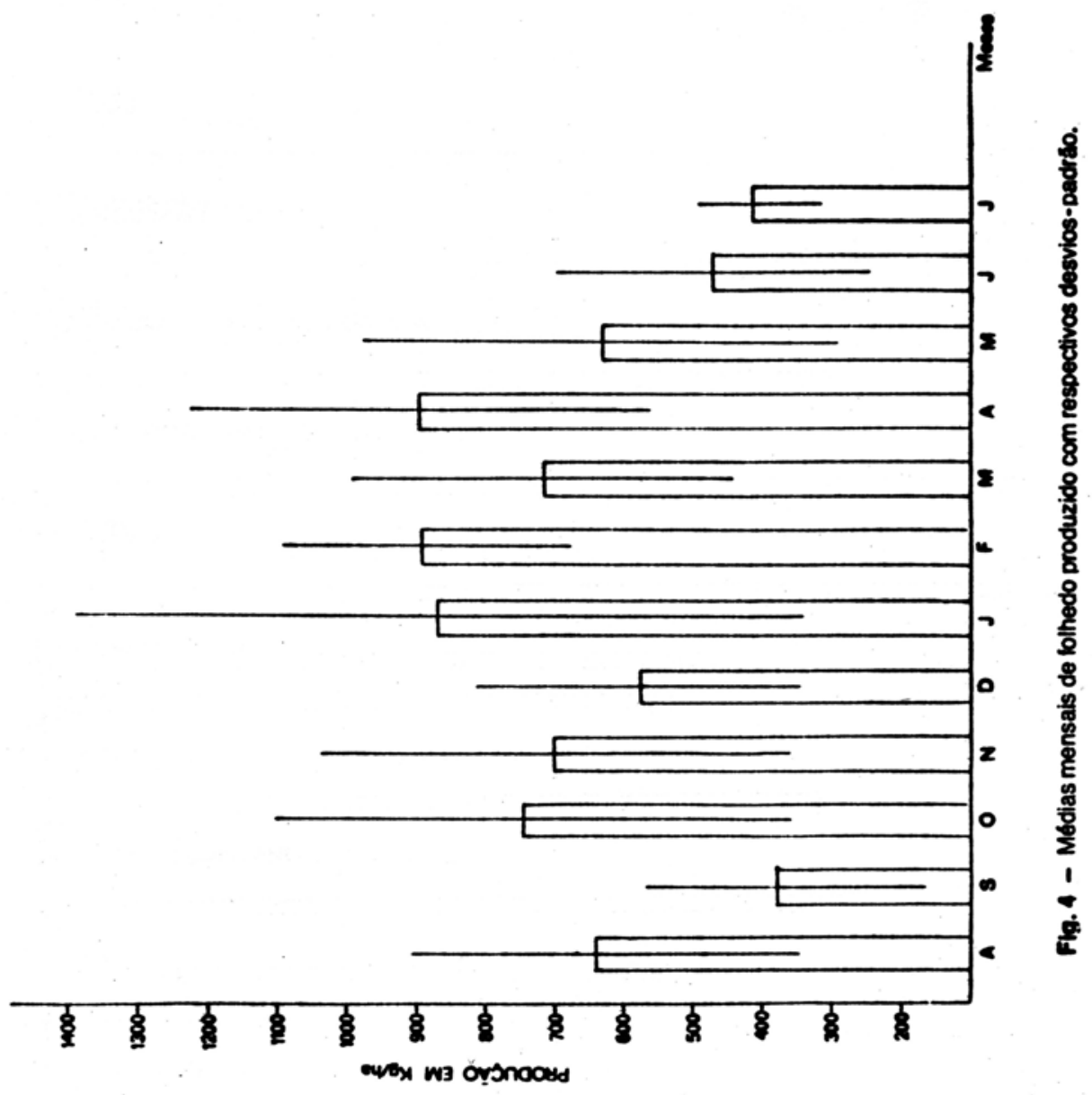




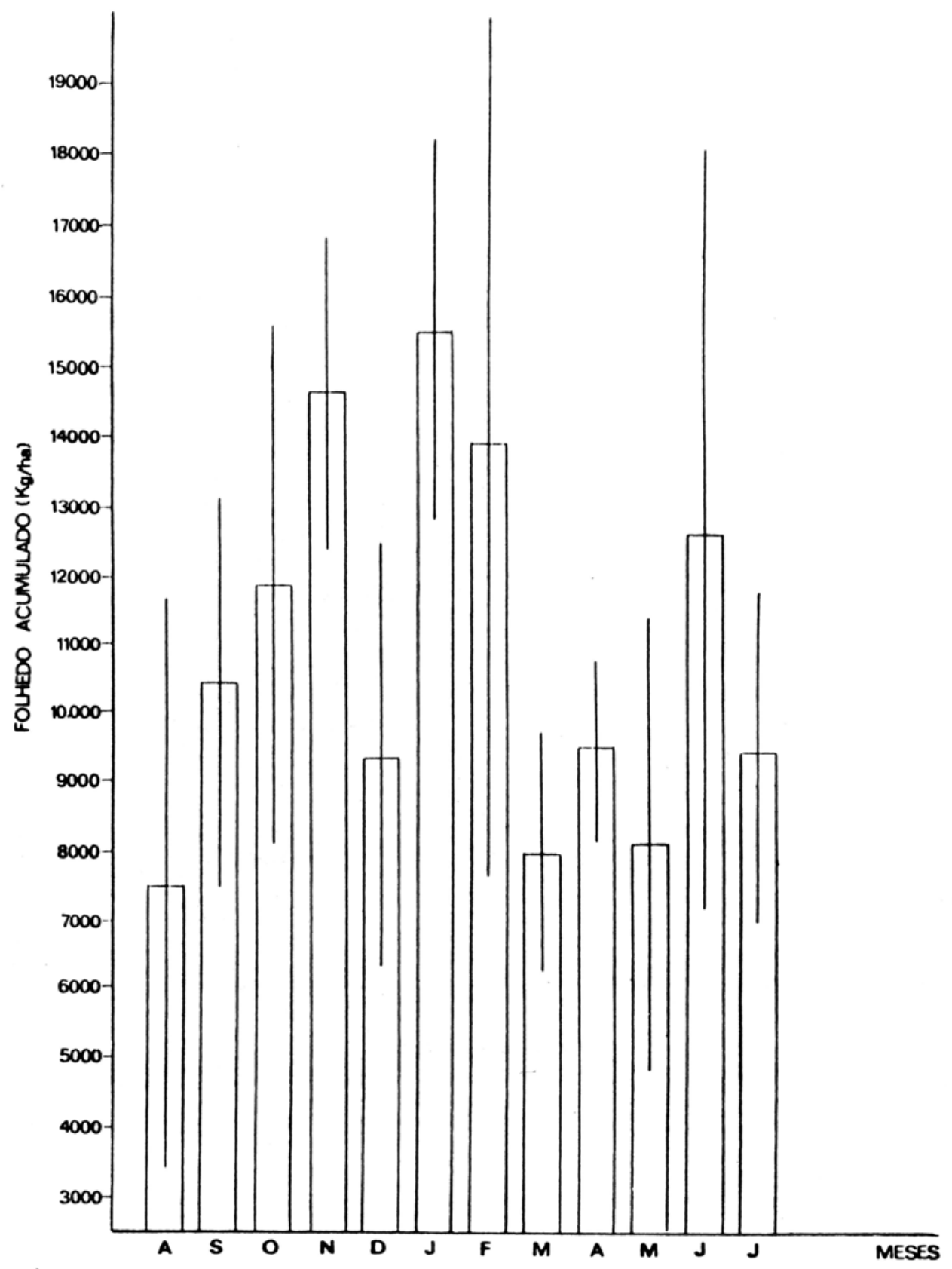

Fig. 5 - Valores mensais médios e os respectivos desvios-padrăo para o folhedo acumulado sobre o solo da área estudada. 


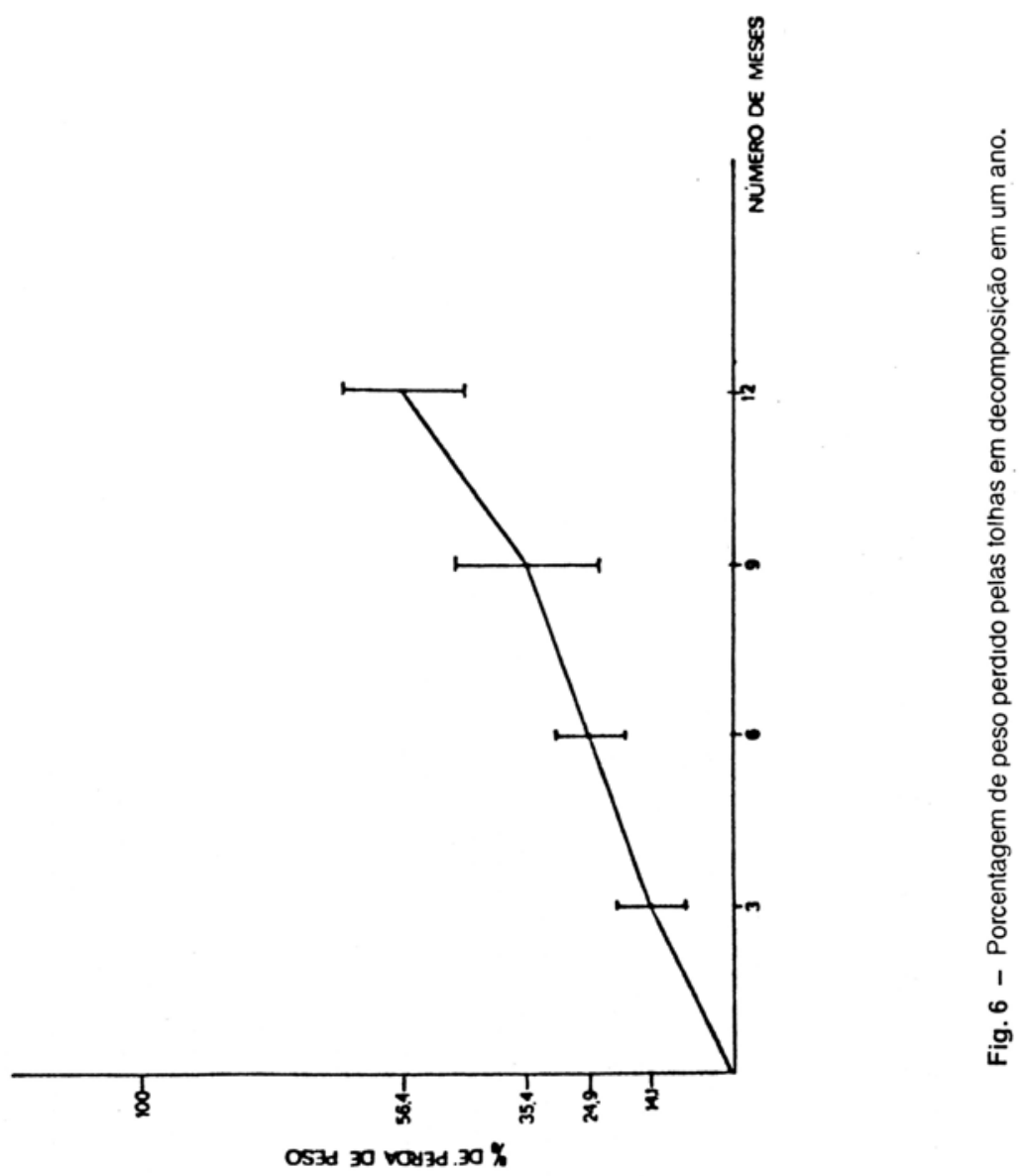

